Check for updates

Cite this: RSC Adv., 2017, 7, 48095

Received 16th August 2017

Accepted 5th October 2017

DOI: $10.1039 / c 7 r a 09048 j$

rsc.li/rsc-advances

\section{A rapid immunochromatographic strip for neutralizing antibodies detection of foot and mouth disease virus serotype 0}

\author{
Suzhen Yang, (D) $\dagger^{a}$ Jifei Yang,,$^{a}$ Yaning Sun, $\dagger^{a}$ Feng Peng, ${ }^{c}$ Shurong Zhang, ${ }^{d}$ \\ Yunchao Liu, ${ }^{a}$ Man Teng, ${ }^{a}$ Dong Zhao, ${ }^{a}$ Shujun Chai ${ }^{a}$ and Gaiping Zhang*be
}

\begin{abstract}
Four peptides were designed and synthesized according to the main FMDV type $O$ vaccine strains in china. Then the synthesized peptides were conjugated to carrier protein. The antigenicity of the artificial antigen was identified by dot-blot assay. Based on the BSA-Pep antigen, a test strip was developed to evaluate the neutralizing antibody of serum samples from swine vaccinated with FMDV type O vaccine rapidly. The BSAPep used as a detector was labeled with colloidal gold. Staphylococcal protein A (SPA) and anti FMDV antibodies were blotted onto the nitrocellulose membrane as the test and control lines, respectively. The strip assay could be performed within $5 \mathrm{~min}$, which did not require any special equipment or skills. Through testing sera against various strains of FMDV type $O$, the sensitivity of the strip was determined to be $95 \%$ (38/40). There were no cross reactions to other virus antibodies, and no cross reactions in different serotypes of FMDV, the test strip can be used to detect FMDV type O antibodies only. The strip results were consistent with those of the existing commercial ELISA kits. In conclusion, the new strip was an acceptable method for surveying FMDV type $\mathrm{O}$ antibody titers in pigs.
\end{abstract}

\section{Introduction}

Foot-and-mouth disease (FMD) is a highly contagious and economically devastating viral disease of cloven-hoofed animals. ${ }^{1,2}$ It is one of the most contagious animal diseases and has adverse socioeconomic effects in the countries where it occurs. ${ }^{3}$ Foot-and-mouth disease virus (FMDV) exists as seven distinct serotypes (O, A, C, Asia 1, SAT 1, SAT 2 and SAT 3) and multiple subtypes. ${ }^{4}$ Of these, type O FMDV is the most variable and therefore is one of the most prevalent FMDV infections in the world. ${ }^{5}$ FMDV is a single-stranded, positivesense virus with an RNA genome of approximately $8.5 \mathrm{~kb}$. The genome encodes a polyprotein precursor that is cleaved to yield four structural proteins (VP4, VP2, VP3 and VP1) and eight non-structural proteins. $^{6}$ The protein VP1 has been shown to be the most important protective antigen, containing critical epitopes

${ }^{a}$ Key Laboratory of Animal Immunology of the Ministry of Agriculture, Henan Provincial Key Laboratory of Animal Immunology, Henan Academy of Agricultural Sciences, Zhengzhou, People's Republic of China

${ }^{b}$ College of Animal Science and Veterinary Medicine, Henan Agricultural University, No. 63 Nongye Road, Zhengzhou 450002, People's Republic of China. E-mail: zhanggaiping2003@163.com

${ }^{c}$ Henan Zhumei Swine Breeding Co., Ltd, Zhumadian, People's Republic of China

${ }^{d}$ Farm Administration Bureau, Department of Agriculture of Henan Province, Zhengzhou, People's Republic of China

'Jiangsu Co-innovation Center for Prevention and Control of Important Animal Infectious Diseases and Zoonoses, Yangzhou, People's Republic of China

$\dagger$ These authors contributed equally to this work. responsible for the induction of neutralizing antibodies ${ }^{7}$ and binding sites of virus receptors. ${ }^{8}$ The prominent G-H loop of the VP1 capsid protein, which spans residues 134-158, has been identified as a major immunogenic site for liciting neutralizing antibodies. ${ }^{9,10}$

Vaccination is a key strategy for the prevention and control of FMD in developing countries. To validate the efficacy of the vaccine, a rapid and simple test is needed in routine field practice to monitor antibody titers against FMDV induced by vaccines. The serological test used most commonly for FMD antibody determination is the virus neutralization test, liquidphase blocking enzyme-linked immunosorbent assay. ${ }^{\mathbf{1 1}}$ Although these assays provide accurate and sensitive detection of anti-FMDV antibodies, they require specialized equipment and technical expertise.

The immunochromatographic strip is a technique in which a cellulose membrane is used as the carrier and a colloidal goldlabeled antigen or antibody is used as the tracer. This technique has several advantages over traditional immunoassays, such as simplicity of the procedure, rapid operation and immediate results, low cost, no requirements for skilled technicians or expensive equipment over the past decade, immunochromatographic strip were applied in many field such as allergies, infectious diseases, environmental contaminants, drugs, fertility, and veterinary. In this study, a simple and rapid strip was developed for the neutralizing antibodies (Ab) detection of foot and mouth disease virus serotype $O$ use the major neutralizing epitope polypeptides. 


\section{Experimental section}

Peptide synthesis and conjugation

Four peptides were designed according to the main FMDV type $\mathrm{O}$ vaccine strains in china, corresponding to the amino acids sequence $140-160$ of VP1 (Table 1). All peptides were synthesized by Gil Biochemical Inc. (Shanghai, China). Peptide purities were $>90 \%$. During synthesis, a cysteine residue was added to the $\mathrm{N}$-terminal of all peptides unless already present, for later conjugation to carrier protein. Peptides were conjugated to a carrier protein of IgG-free BSA (Sigma, St. Louis, MO) using a hetero-bifunctional crosslinker Sulfo-SMCC (Pierce, Rockford, IL) according to the manufacturer's instructions. The peptides which coupled with BSA (BSA-Pep) were using as antigen against FMDV serotype $\mathrm{O}$, and preparing FMDV serotype $\mathrm{O}$ neutralizing antibodies detection strip.

\section{Peptide identification}

A dot-blot assay was developed to identify the binding of synthesized peptides to positive sera of FMDV type O. The BSA coupled peptides (BSA-pep) were blotted onto nitrocellulose membranes (Millipore, Billerica, MA) at $0.3 \mu \mathrm{g}$ per dot, respectively; $0.3 \mu \mathrm{g}$ per dot of the carrier protein BSA were used as negative. After air drying, the membrane was blocked with $0.2 \%$ gelatin in $0.01 \mathrm{M}$ PBS (pH 7.2) that contained $0.05 \%$ tween 20 (PBST) at $37^{\circ} \mathrm{C}$ for $1 \mathrm{~h}$; the membrane was incubated with the corresponding strain FMDV positive sera; then, horseradish peroxidase-conjugated goat anti pig IgG (HRP-IgG) in PBST containing $0.2 \%$ gelatin at $37{ }^{\circ} \mathrm{C}$ for $1 \mathrm{~h}$ followed by thorough washing with PBST. Color was developed using a 3-amino-9ethylcarbazole staining kit according to the manufacturer's instructions.

\section{Preparation of colloidal gold-BSA-Pep probe and test strip}

Colloidal gold was prepared by the modified method reported earlier, ${ }^{12}$ whose mean particle diameter was about $20 \mathrm{~nm}$. The $\mathrm{pH}$ of colloidal gold solution was adjusted to 8.0 using $0.2 \mathrm{~mol} \mathrm{~L}^{-1} \mathrm{~K}_{2} \mathrm{CO}_{3}$ aqueous.

Four BSA-Pep antigens were diluted to $2 \mathrm{mg} \mathrm{mL}^{-1}$ in normal saline and mixing with the same volume. Then gold-labeled antigen (BSA-Pep) was prepared according to a previously described method. ${ }^{13}$

The test strip was composed of a plastic packing, a NC membrane and three pads (sample, probe and absorbent pads) (Fig. 1A). The NC membrane $(20 \mathrm{~mm} \times 300 \mathrm{~mm})$ was spotted with

Table 1 Synthetic peptides sequence of foot-and-mouth disease virus serotype $\mathrm{O}$

\begin{tabular}{lll}
\hline Peptide ID & Amino acid sequence & Reference stain \\
\hline Pep 1 & CSTNNVRGDLQVLAQKAERALP & O/GX/09-7 \\
Pep 2 & CRVSNVRGDLQVLAQKAERALP & O/HN/CHA/93 \\
Pep 3 & CSTNNVRGDLQVLAQKAERTLP & O/TAW/97 \\
Pep 4 & CSLTNVRGDLQVLAQKAARPLP & O/MYA/98
\end{tabular}

Staphylococal Protein A (SPA) and FMDV VP1 monoclone antibody IgG as the test line ( $\mathrm{T}$ line) and control line (C line), respectively, leaving a $0.5 \mathrm{~cm}$ space between the two lines. The reagents were applied as dots at $1 \mu \mathrm{L} \mathrm{cm}^{-1}$. After drying for $1 \mathrm{~h}$ at $45^{\circ} \mathrm{C}$, sealed and stored under dry condition. The sample pad $(15 \mathrm{~mm} \times 300$ $\mathrm{mm}$ ) was saturated with $0.1 \mathrm{~mol} \mathrm{~L}^{-1}$ Tris-HCl ( $\mathrm{pH}$ 8.0), then dried at $45{ }^{\circ} \mathrm{C}$ for $4 \mathrm{~h}$. The probe pad was saturated with $8 \mu \mathrm{L} \mathrm{cm}^{-1}$ of BSA-Pep-gold conjugate solution and dried at $37^{\circ} \mathrm{C}$ for $40 \mathrm{~min}$. The adsorbent pad was cut into dimensions of $20 \mathrm{~mm} \times 300 \mathrm{~mm}$. The sample pad, probe pad, absorbent pad and NC membrane were pasted on the plastic packing sequentially with 1-2 mm overlap. The master card was cut into $3 \mathrm{~mm}$ wide strips, then the strips were sealed and stored at $4{ }^{\circ} \mathrm{C}$.

\section{Test procedure and principle}

In the detection test, $100 \mu \mathrm{L}$ serum sample diluted 200 -fold with normal saline was spotted on the sample pad, which could flow towards the absorbent pad along the NC membrane. Five to $10 \mathrm{~min}$ later, for a positive sample, the gold-BSA-Pep conjugate would bind to the IgG of FMDV antibodies, then IgG trapped by SPA on the test line which could turn red obviously. As to a negative sample, IgG in serum could be trapped by SPA, but it cannot bind to the gold-BSA-Pep conjugate, there is no any color on the test line. Once the control lines became red, the strip and the test procedure were correct. Otherwise, when the control line was colorless, the strip might be invalid or the test procedure might be improper. The test should be repeated using new strips in this case (Fig. 1B).

\section{Identification of the test strip}

Sensitivity of the strip. A series FMDV type O antibody strong or weak positive serum was used to evaluate the sensitivity of FMDV neutralizing Ab strips. The sera groups were described as

A

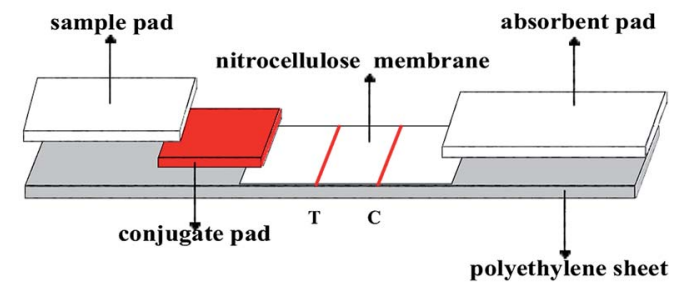

B
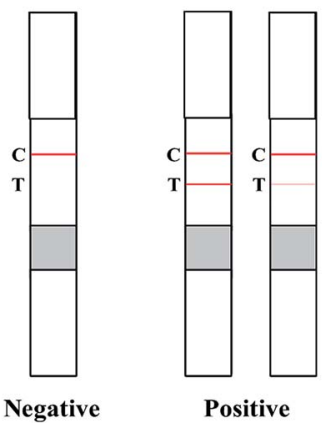

Positive

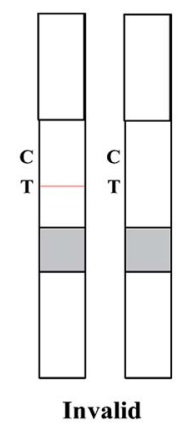

Fig. 1 Structure diagram of the test strip for FMDV type O neutralizing antibody detection (A), and the expected pattern of visual results in the test procedure (B). 
follow: (I) a total of 20 serum samples from FMDV type $\mathrm{O}$ vaccinated swine, including vaccinated swine against FMDV O/MYA98/ $\mathrm{BY} / 2010$ strain $(n=8)$, immunized swine against FMDV O/GX/097 and $\mathrm{O} / \mathrm{XJ} / 10-11$ strain $(n=6)$, immunized swine against FMDV $\mathrm{O} / \mathrm{ZK} / 93$ and $\mathrm{OR} / 80$ strain $(n=6)$; (II) serum samples from infected swine by FMDV O/MYA98 $(n=5)$; (III) serum samples obtained from field swine farm which immunized by FMDV type $O$ vaccine $(n=15)$. All of this 40 serum samples showed different levels of antibody titers ( $\geq 1: 64)$ against FMDV type $O$ by a commercial liquid phase blocking ELISA (LPB-ELISA). The detection limit of the strip was tested with serially diluted FMDVantibody positive reference sera (come from national foot and mouth disease reference laboratory). Each serum sample was tested in triplicate by strip assay. The strip assay procedures is as follows: dip the strip sample end into $1: 100$ diluted serum for 10-20 s, remove the strip and lay flat, observe the result within 5$10 \mathrm{~min}$. When both of the test and control lines turned red, it indicated that the sample was FMDV type $\mathrm{O}$ antibody positive. If only the control line appeared red, the sample was considered as FMDV type $\mathrm{O}$ antibody negative. The serum samples were diluted with $0.9 \%$ normal saline (NS).

Specificity of the strip. The specificity of the new strip was determined with 43 reference serum samples, including FMDV standard positive sera $(n=10)$ and FMDV negative sera $(n=10)$ as well as 23 positive sera against a variety of other viruses (PRRSV, PRV, CSFV, PCV2, JEV, SIV).

Cross-reaction in different serotypes. To evaluate the crossreactivity of the test strip, the sera of serotype $\mathrm{O}$, A and Asia1 were used, 3 standard sera of each serotype were selected, and the negative serum was as used as control. All sera were tested according to the test procedure described above.

Comparison with commercial ELISAs. A total of 356 clinical swine serum samples were tested in triplicate by using three different batches of strip, these sera were also screened for antibodies of FMDV using two kinds of commercial liquid phase blocking ELISA kits (LPB ELISA come from the Lanzhou Veterinary Research Institution in China (LVRI) and VDPro® FMDV antibody ELISA (MEDIAN, Korea)), simultaneously. The strip assay was performed as described above and the ELISAs were done according to the manufacturer's description.

The early neutralizing antibody response in FMDV type $O$ immunized animal. Ten piglets were tagged and vaccinated with FMDV type $O$ vaccines, initial immunization was performed at the age of $55 \mathrm{~d}$, and the second immunization was performed after $28 \mathrm{~d}$. Blood was collected from each piglet at different times. All collected sera were tested by the new strip and commercial LPB ELISA, separately.

\section{Ethics statement}

The animal experiments were carried out according to the Animal Experiment Committee of Henan Academy of Agricultural Sciences (Approval number SYXK 2014-0007). All animals received humane care in compliance with good animal practice according to the animal ethics procedures and guidelines of China.

\section{Results and discussion}

\section{Antigen preparation}

The products of synthesized peptides coupled with BSA were identified by SDS-PAGE. The molecular weight in SDS-PAGE of BSA-Pep were different from BSA. Compared with BSA control sample, the molecular weights of all four conjugates were increased, as shown by PAGE (Fig. 2), indicating that the peptides were successfully linked to the carrier proteins.

\section{Antigen identification}

The antigenic activity of peptides was analyzed by using the corresponding strain FMDV positive sera (antibody titers: $\geq: 128$ determined by LPB ELISA) and negative serum sample by dot-blot assay. If antigen reacted to the serum, there will display a red color on the dot-blot membrane, the color density of dot-blot results was scanned with a Bio-Dot TSR3000 membrane strip reader. The results showed that all peptides reacted to their positive sera in different degree and did not react with negative animal serum (Fig. 3). To detect the antibodies against different virus strains of FMDV vaccine, all the four artificial antigen were mixed equally, and used to develop the neutralizing $\mathrm{Ab}$ test strip.

\section{Sensitivity of the strip}

To evaluate the sensitivity of FMDV neutralizing Ab strip for serological detection of FMDV, a series serum of vaccinated animals with different strain, infected animals, as well as field sera were tested with FMDV neutralizing Ab strip assay as described above. The results demonstrated that the strip assay

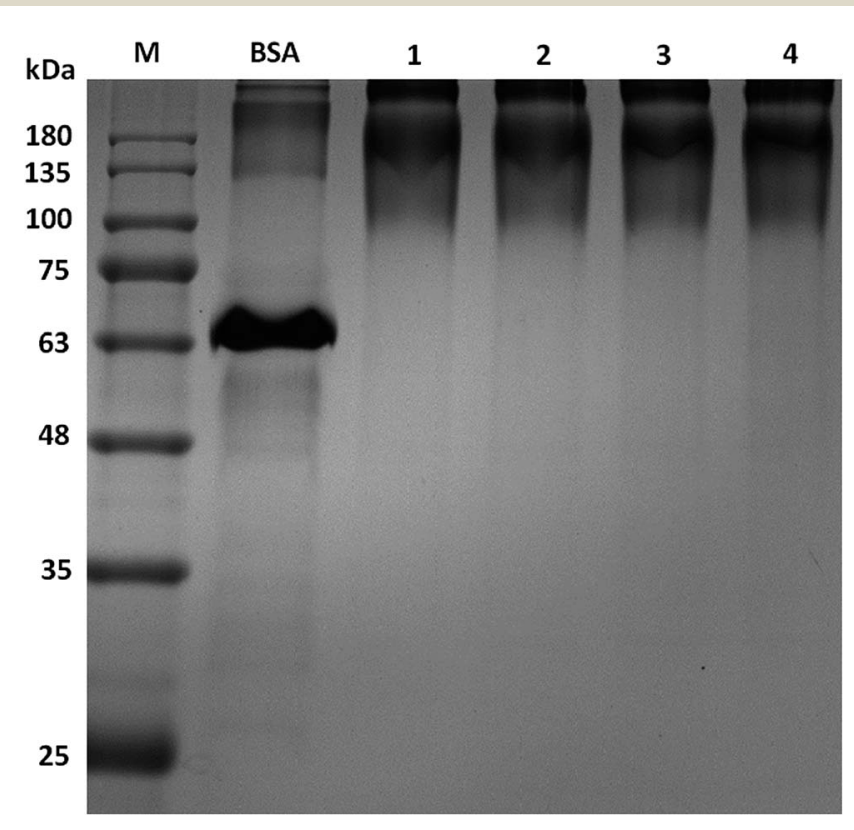

Fig. 2 SDS-PAGE analysis of peptides conjugates and carrier proteins. The protein bands were stained with Coomassie blue. Lanes: $M$, the protein standards with their molecular masses in kilodaltons shown on the left; BSA, carrier protein BSA; 1-4, conjugate BSA-Pep, The sequence of peptides was Pep1, Pep2, Pep3, Pep4. The migration rates of all conjugates were less than BSA. 


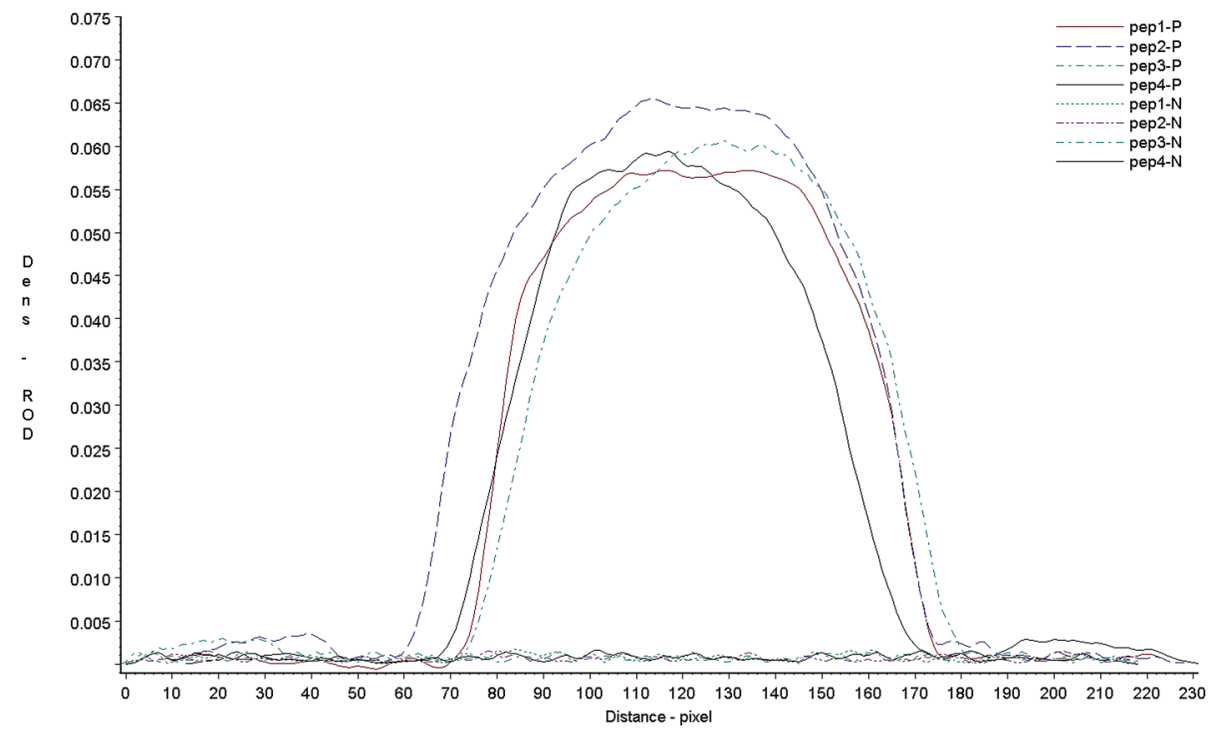

Fig. 3 Relative optical density (ROD) curves of chromogenic results on the dot-blot membrane. Pep1-P, Pep2-P, Pep3-P, Pep4-P means the four BSA-Peps response results to the corresponding strain FMDV positive sera. The stronger the antigen and antibody response, the higher the curve of the optical density. Pep1-N, Pep2-N, Pep3-N, Pep4-N means the four BSA-Peps response results to FMDV negative serum, there have almost no any curve.

was sensitive for FMDV antibody detection with a sensitivity of 95\% (38/40) (Table 2). The detection limit of the strip was tested with serially diluted FMDV-antibody positive reference serum, which was $1: 1600$, the LPB ELISA titer of this serum was $1: 128$. It meant that a red band could be seen clearly at the test line position when the dilution of the serum sample was $1: 1600$ or less. The same titer was found by using the ELISA (date not shown).

\section{Specificity of the strip}

The specificity of the new strip was determined with 43 reference serum samples, including 10 FMDV standard positive sera and 10 negative sera as well as 23 positive sera against a variety of other viruses (PRRSV, PRV, CSFV, PCV2, JEV, SIV). Fig. 4 showed that a positive result appeared with two visible red bands at the test and control line positions, and a negative result appeared with only one red band at the control line position on the strip. The results from swine serum samples were summarized in Table 3. All of the anti-FMDV standard serum samples were found to be positive, while the other serum samples were shown to be negative. The results indicated that there was no cross-reactivity with antibodies against any other virus, the specificity of the new strip was $100 \%$, proving that the strip was specific for detecting FMDV antibody.

\section{Cross-reaction in different FMDV serotypes}

Cross-reaction of the test strip with anti-bodies against different serotypes of FMD was determined by measuring the test responses to type $\mathrm{O}$, A, Asia1 positive samples. FMD negative serum was included as a control sample. The strip control and

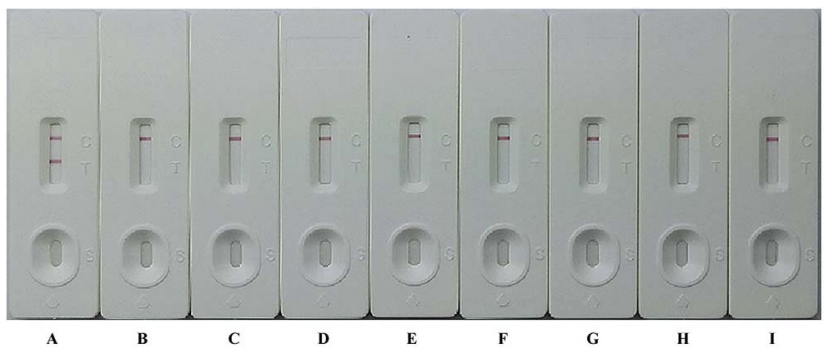

Fig. 4 The specificity of FMDV antibody detection by the test strip. Note: A: pig anti-FMDV type O serum, B: pig FMDV negative serum, C: pig anti-PRRSV serum, D: pig anti-PRV serum, E: pig anti-CSFV serum, F: pig anti-PCV2 serum, G: pig anti-JEV serum, H: pig anti-SIV serum, I: normal saline.

Table 2 The sensitivity of the test strip with different swine serum samples

\begin{tabular}{|c|c|c|c|c|}
\hline Serum ID & Serum background & Vaccine strains & Strip positive & Total \\
\hline P1-8 & Immunized & O/MYA98/BY/2010 & 8 & 8 \\
\hline P9-14 & Immunized & $\mathrm{O} / \mathrm{GX} / 09-7+\mathrm{O} / \mathrm{XJ} / 10-11$ & 6 & 6 \\
\hline P21-25 & Infected & O/MYA98 & 6 & 6 \\
\hline P26-40 & Field immunized swine & O/MYA98/BY/2010 & 13 & 15 \\
\hline
\end{tabular}


Table 3 The specificity of the test strip with reference swine serum samples

\begin{tabular}{llll}
\hline & \multicolumn{2}{l}{ Results from strip assay } & \\
\cline { 2 - 3 } Antibody status & Positive & Negative & Total \\
\hline FMDV positive & 10 & 0 & 10 \\
FMDV negative & 0 & 10 & 10 \\
PRRSV & 0 & 4 & 4 \\
PRV & 0 & 4 & 4 \\
CSFV & 0 & 4 & 4 \\
PCV2 & 0 & 4 & 4 \\
JEV & 0 & 4 & 4 \\
SIV & 0 & 3 & 3 \\
\hline
\end{tabular}

test lines were scanned with a Bio-Dot TSR3000 membrane strip reader (Fig. 5), the results showed that when test the type $\mathrm{O}$ antisera all strips gave a clear positive. However, other anti-sera gave no visible line in the test zone, just like the negative sera. There was no any cross-reaction in different serotypes of FMDV, the test strip can be used to detect FMDV type O antibodies only. The scanned results of A and Asia1 sera were basically the same as negative sera.

\section{Accuracy of the test strip}

To determine the accuracy of the test strip, a comparison between the strip and two commercial ELISA kits was performed by using 356 clinical serum samples from several pig farms. The test strip provided clear positive or negative results with these field samples. Samples with ambiguous results were retested. If the test provided another ambiguous result, the sample was considered as negative. The test strip and the two
Table 4 Comparison of the test strip with two commercial ELISA kits ${ }^{a}$

\begin{tabular}{|c|c|c|c|c|}
\hline & \multicolumn{2}{|l|}{ LPB ELISA kit } & \multicolumn{2}{|c|}{$\begin{array}{l}\text { VDPro® FMDV } \\
\text { antibody ELISA kit }\end{array}$} \\
\hline & $\begin{array}{l}\text { Positive } \\
\text { (titer } \geq 1: 64 \text { ) }\end{array}$ & $\begin{array}{l}\text { Negative } \\
\text { (titer }<1: 64)\end{array}$ & $\begin{array}{l}\text { Positive } \\
(\mathrm{S} / \mathrm{P}<0.6)\end{array}$ & $\begin{array}{l}\text { Negative } \\
(\mathrm{S} / \mathrm{P} \geq 0.6)\end{array}$ \\
\hline \multicolumn{5}{|c|}{ Strip assay } \\
\hline Positive & 301 & 25 & 296 & 22 \\
\hline Negative & 3 & 27 & 8 & 30 \\
\hline
\end{tabular}

commercial ELISA kits (LVRI LPB ELISA and VDPro ${ }^{\circledR}$ FMDV antibody ELISA) provided similar results (Table 4). The agreement of the test strip with the LVRI LPB ELISA and VDPro® FMDV antibody ELISA was 92.1\% (328/356) and 91.6\% (3.26/ 356), respectively. This indicated that the three test methods show good correspondence.

\section{The early neutralizing antibody response in FMDV type $O$ immunized animal}

Ten vaccinated piglets sera were collected at different time as described above, all collected sera were tested by the new strip and commercial LPB ELISA, separately (Fig. 6). The strip tested results showed the positive antibody appeared after immunized 8 days, then the antibody increased rapidly, and reached the peak at 28 days, however, there were still two pigs no antibody response nearly. And this result was similar to the result of ELISA.

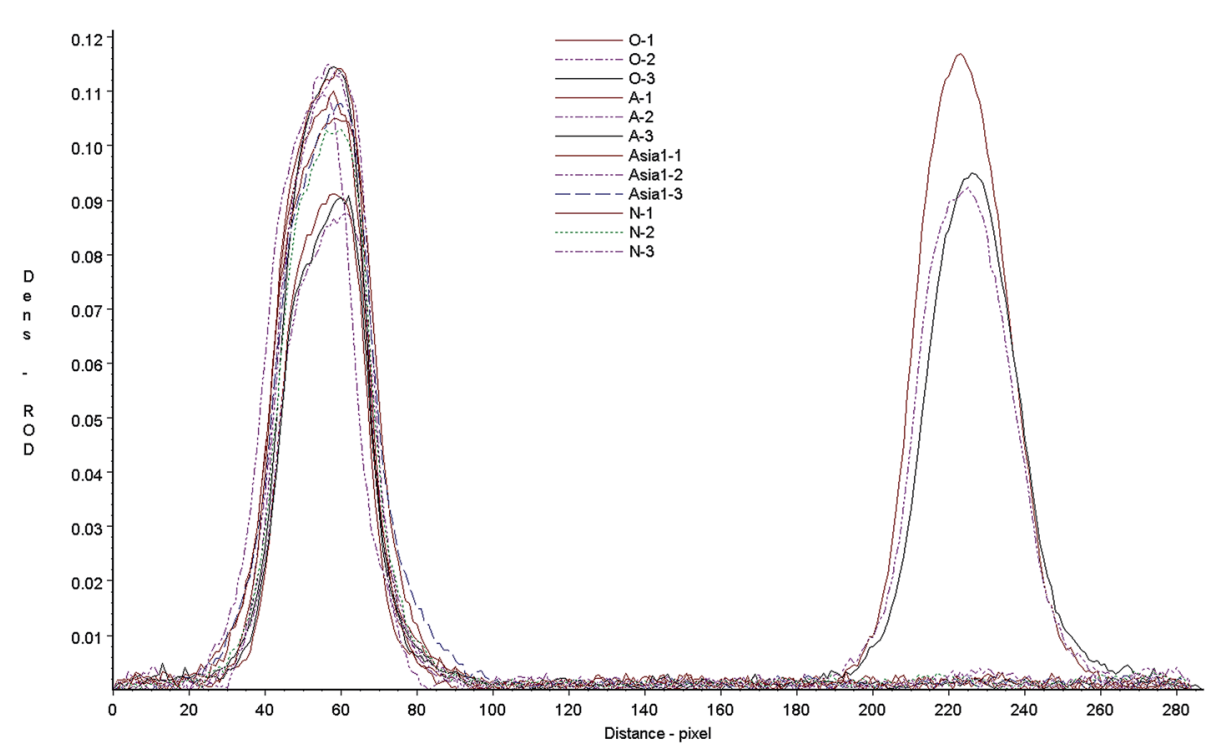

Fig. 5 Relative optical density (ROD) curves of standard samples. Positive sera of FMDV serotype O, A, Asia1 and negative control. The left peaks: scanned results of control lines, the right peaks: scanned results of test lines. O-1, O-2, O-3: 3 FMDV serotype O positive sera, A-1, A-2, A-3: 3 FMDV serotype A positive sera, Asia1-1, Asia1-2, Asia1-3: 3 FMDV serotype O positive sera, N-1, N-2, N-3: FMDV serotype O negative sera. All of the 12 sera gave visible peaks on the control line, only 3 FMDV type $O$ sera give peaks on the control line, and the test results of A and Asia1 sera were basically the same as negative sera. 

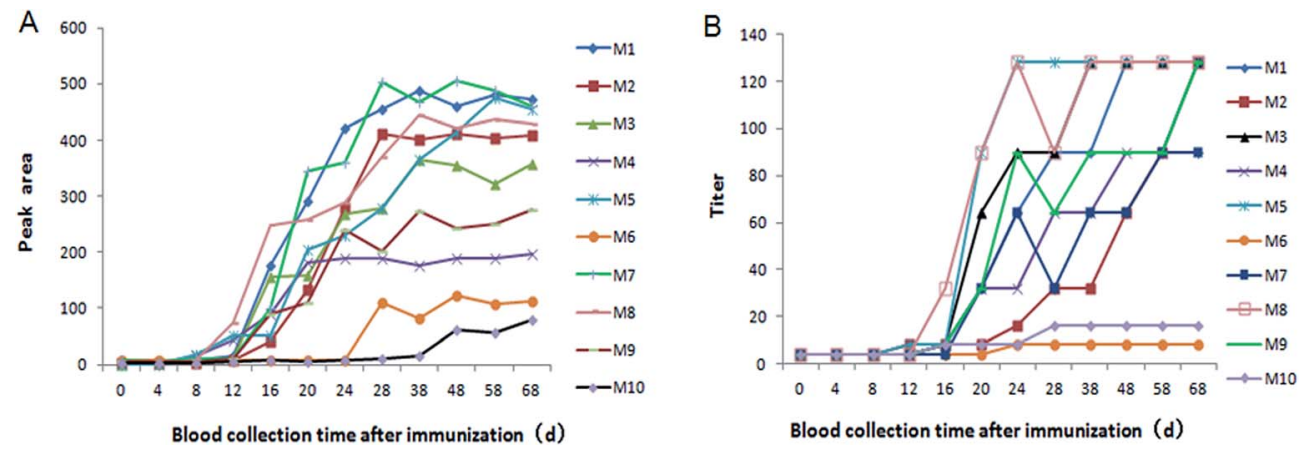

Fig. 6 The early neutralizing antibody response in FMDV type $O$ immunized animal (A) The early neutralizing antibody response in FMDV type $O$ immunized detected by strip. (B) The early neutralizing antibody response in FMDV type O immunized detected by LPB ELISA kit.

\section{Conclusions}

FMD exists as seven major serotypes and numerous subtypes, the practical implication of which is that single or multiple vaccinations with one serotype do not confer protection against the other six serotypes. The VP1 protein of FMDV is the key protective antigen which induces a strong immunologic response including neutralizing antibodies. This makes it a highly suitable target antigen in developing serological tests. However, the VP1 protein used in these test procedures were primarily produced in expression systems by using either bacteria or baculovirus, and nonspecific reactions can be caused by the presence of antibodies in test animals against these expression vector antigens. It is reported that the prominent G-H loop of the VP1 capsid protein, which spans residues 134-158, has been identified as a major immunogenic site for liciting neutralizing antibodies, some FMDV peptide vaccines were improved use this $\mathrm{G}-\mathrm{H}$ loop, ${ }^{\mathbf{1 4}, 15}$ In this study, four peptides were designed according to the main FMDV type $\mathrm{O}$ vaccine strains in china and synthesized by solid-phase peptide synthesis, then peptides were coupled with carrier protein as antigen improved a rapid antibody test strip. The strip assay could be performed within $5 \mathrm{~min}$, which did not require any special equipment or skills. This strip can qualitative detection FMDV serotype $\mathrm{O}$ antibody, the disadvantages of the strip is it could not precisely quantify the antibody level, the quantitative data of the strip was acquired according to the control ELISA (liquid phase blocking ELISA), the ELISA titer $\geq 1: 64$ means the antibody was enough to resist the FMD type $\mathrm{O}$ virus, so during the research and development of the strip, the test results were adjusted according the ELISA results. When the ELISA titer $\geq 1: 64$, the strip give a positive result, when the ELISA titer $<1: 64$ the strip give a negative result. The positive results of the strip means the antibody was enough to resist the FMD type $O$ virus. Through testing sera against various strains of FMDV type $\mathrm{O}$, the sensitivity of the strip was determined to be $95 \%(38 / 40)$ and the specificity was $100 \%$, no any cross reactions to other virus antibody. Then, there was no any cross reaction in different serotypes of FMDV, the test strip can be used to detect FMDV type $\mathrm{O}$ antibodies only. The strip results were consistent with those of the existing commercial ELISA kits. In conclusion, the new strip was an acceptable method for surveying FMDV type $\mathrm{O}$ antibody titers in pigs.

A typical characteristic of FMDV is highly variable, there is no or less cross-reactions in different serotype even in different subtype. In China, virus strains of FMDV vaccine mainly including O/MYA98/BY/2010, O/GX/09-7, O/XJ/10-11, O/ZK/93, $\mathrm{OR} / 80$, one kind of antigen will not react well to the antibody immunized with different virus strains, therefore, four peptides were designed according to the five vaccine strains, and peptides antigen were mixed equally.

In China, ELISA is the gold standard for FMDV antibody determination, however this assay requires the blood/serum samples to be sent to testing laboratories with extra costs, labor and time. Colloidal gold as an indicator has been used in electron microscopy, light microscopy, and immunoblotting for a long time. Colloidal gold conjugates of both antibodies and antigens have been used as detection reagents for testing animal diseases and drug residues. ${ }^{16,17}$ The strip can solve the above problems with immediate assessment of the vaccination on the spot. In addition, the strip can be stored easily with low cost. The weakness is that the FMDV antibody strip assay cannot differentiate infected animals from vaccinated animals, our team had developed a kind of strip which can differentiate FMDV infected animals from vaccinated animals, in the nearly future a new strip will be established simultaneous evaluate the antibody level of FMDV immunized animal and differentiate FMDV infected animals from vaccinated animals in one strip.

\section{Conflicts of interest}

There are no conflicts to declare.

\section{Acknowledgements}

This work was supported by the National Key Research and Development Program of China (no. 2016YFD0501503 and no. 2016 YFD050707), China Agriculture Research System (CARS-36).

\section{References}

1 H. L. Bachrach, Beltsville Symposia in Agricultural Research, Virology in Agriculture, 1977. 
2 H. G. Pereira, Dev. Biol. Stand., 1976, 35, 167-174.

3 R. P. Kitching, Vaccine, 1999, 13-14, 1772-1774.

4 N. J. Knowles and A. R. Samuel, Virus Res., 2003, 1, 65-80.

5 W. Liang, G. Zhou, W. Liu, B. Yang, C. Li, H. Wang, D. Yang, W. Ma and Y. Li, Arch. Virol., 2016, 10, 2705.

6 E. Domingo, E. Baranowski, C. Escarmís and F. Sobrino, Comp Immunol Microbiol Infect Dis., 2002, 5-6, 297-308.

7 D. Haydon, S. Lea, L. Fry, N. Knowles, A. R. Samuel, D. Stuart and M. E. Woolhouse, J. Mol. Evol., 1998, 4, 465-475.

8 J. H. Wang, C. M. Liang, J. M. Peng, J. J. Shieh, M. H. Jong, Y. L. Lin, M. Sieber and S. M. Liang, Vaccine, 2003, 25-26, 3721-3729.

9 N. Verdaguer, M. G. Mateu, D. Andreu, E. Giralt, E. Domingo and I. Fita, EMBO J., 1995, 8, 1690-1696.

10 J. L. Bittle, R. A. Houghten, H. Alexander, T. M. Shinnick, J. G. Sutcliffe, R. A. Lerner, D. J. Rowlands and F. Brown, Nature, 1982, 5869, 30-33.

11 J. P. Araujo, H. J. Montassier and A. A. Pinto, Am. J. Vet. Res., 1996, 6, 840-843.
12 S. Yang, J. Yang, G. Zhang, X. Wang, S. Qiao, D. Zhao, Y. Zhi, X. Li, G. Xing, J. Luo, J. Fan and D. Bao, J. Virol. Methods, 2010, 2, 139-144.

13 S. Yang, J. Yang, G. Zhang, S. Qiao, X. Wang, D. Zhao, X. Li, R. Deng, A. Zhi, L. You, S. Chai and M. Teng, J. Vet. Diagn. Invest., 2010, 3, 412-415.

14 H. B. Lee, D. C. Piao, J. Y. Lee, J. Y. Choi, J. D. Bok, C. S. Cho, S. K. Kang and Y. J. Choi, Microb. Cell Fact., 2017, 1, 33.

15 Y. Cao, Z. Lu, Y. Li, P. Sun, D. Li, P. Li, X. Bai, Y. Fu, H. Bao, C. Zhou, B. Xie, Y. Chen and Z. Liu, Antiviral Res., 2013, 2, 145-153.

16 G. Zhang, X. Wang, A. Zhi, Y. Bao, Y. Yang, M. Qu, J. Luo, Q. Li, J. Guo, Z. Wang, J. Yang, G. Xing, S. Chai, T. Shi and Q. Liu, Food Addit. Contam., Part A, 2008, 4, 413-423.

17 G. P. Zhang, J. Q. Guo, X. N. Wang, J. X. Yang, Y. Y. Yang, Q. M. Li, X. W. Li, R. G. Deng, Z. J. Xiao, J. F. Yang, G. X. Xing and D. Zhao, Vet. Parasitol., 2006, 3-4, 286-293. 